\title{
Efeitos cardiorrespiratórios do butorfanol em cães pré-tratados ou não pela levomepromazina
}

\author{
Cardiopulmonary effects of butorphanol in dogs treated with levomepromazine \\ Paulo Sérgio Patto dos Santos ${ }^{1}$ Newton Nunes ${ }^{2}$ Márlis Langenegger de Rezende ${ }^{3}$ \\ Almir Pereira de Souza ${ }^{4}$ Celina Tie Nishimori ${ }^{3}$ Danielli Parrilha de Paula ${ }^{3}$ \\ Marcus Okamura ${ }^{5}$
}

\section{RESUMO}

Objetivou-se com este experimento avaliar os efeitos do butorfanol precedido ou não pela levomepromazina sobre a freqüência cardíaca (FC), as pressões arteriais sistólica, diastólica e média (PAS, PAD e PAM, respectivamente), a freqüência respiratória (f), a concentração de dióxido de carbono ao final da expiração (ETCO ${ }_{2}$ ), a saturação da oxihemoglobina $\left(\mathrm{SpO}_{2}\right)$, o volume corrente (VC) e o volume minuto (VM), em cães. Para tal, foram empregados vinte animais adultos, clinicamente saudáveis, distribuídos igualmente em dois grupos (GC e GL). Ao GC administrou-se solução salina a 0,9\% (Controle), no volume de $0,2 \mathrm{~mL} \mathrm{~kg}^{-1}$, pela via intravenosa (IV). Decorridos 15 minutos, administrouse butorfanol na dose de $0,3 \mathrm{mg} \mathrm{kg}^{-1}$ pela mesma via. Aos animais do GL foi adotada a mesma metodologia, porém substituindo-se a solução salina pela levomepromazina na dose de $1 \mathrm{mg} \mathrm{kg}^{-1}$. As medidas das variáveis cardiorrespiratórias iniciaram-se imediatamente antes da aplicação dos fármacos (M1). Novas mensurações foram realizadas 15 minutos após a administração da solução salina a 0,9\% ou levomepromazina (M2) e 10 minutos após a administração de butorfanol (M3). As demais colheitas foram realizadas a intervalos de 10 minutos, durante 30 minutos (M4, M5 e M6, respectivamente). Os dados numéricos colhidos foram submetidos à Análise de Variância (ANOVA), seguida pelo teste de Tukey $(p<0,05)$ para as comparações das médias. O emprego do butorfanol promoveu diminuição significativa das freqüências cardíaca e respiratória e do volume minuto no grupo previamente tratado pela levomepromazina; entretanto, essas alterações foram discretas, não comprometendo os demais parâmetros circulatórios e respiratórios.

Palavras-chave: cardiopulmonar, cães, levomepromazina, butorfanol.

\begin{abstract}
This work was aimed at evaluating the effects of the butorphanol in dogs preceded or not buy levomepromazine on heart rate (HR), systolic, diastolic and mean arterial pressure (SAP, DAP and MAP, respectively), respiratory rate (RR), end tidal $\mathrm{CO}_{2}\left(\mathrm{ETCO}_{2}\right)$, oxyhemoglobin saturation $\left(\mathrm{SpO}_{2}\right)$, minute volume (MV) and tidal volume (TV). Twenty adult animals, clinically health were randomly distributed in two groups with ten animals each one (CG and LG). The first one received intravenous administration (IV) of $0.2 \mathrm{~mL} \mathrm{~kg}^{-1}$ of saline solution at $0.9 \%$ (control). After 15 minutes, $0.3 \mathrm{mg} \mathrm{kg}^{-1}$ of butorphanol was administrated by the same way. The same methodology was adopted to the GL animals, however, the saline solution at $0.9 \%$ was substituted to $1 \mathrm{mg} \mathrm{kg}^{-1}$ of levomepromazine. The cardiorespiratory parameters were measured before the administration of the drugs (M1). New measurements were carried 15 minutes after saline or levomepromazine administration (M2), and 10 minutes after butorphanol administration (M3). The next M4, M5 and M6 were carried out through at intervals of 10 minutes during 30 minutes. The numeric data were submitted to the Analysis of Variance (ANOVA) followed by Tukey test $(p<0.05)$ for the comparisons of the averages. The butorfanol promoted significative decreasing on cardiac and respiratory rate and reduction on minute volume after administration of levomepromazine. However these changes were discreet and did not produce depression on other circulatory and breathing parameters.
\end{abstract}

Key words: cardiopulmonary, dogs, levomepromazine, butorphanol.

${ }^{1}$ Curso de Medicina Veterinária, Centro Universitário Barão de Mauá, Ribeirão Preto, SP, Brasil. E-mail: pspatto@itelefonica.com.br. Autor para correspondência.

²Departamento de Clínica e Cirurgia Veterinária, Faculdade de Ciências Agrárias e Veterinárias (FCAV), UNESP, Jaboticabal, SP, Brasil.

${ }^{3}$ Programa de Pós-graduação em Cirurgia Veterinária, FCAV, UNESP, Jaboticabal, SP, Brasil.

${ }^{4}$ Universidade Federal de Campina Grande, Campus de Patos, PB, Brasil.

${ }^{5}$ Autônomo. 


\section{INTRODUÇÃO}

O profissional que se dedica à prática clínicocirúrgica freqüentemente necessita empregar, para contenção farmacológica e potenciação do efeito de agentes anestésicos, fármacos que promovam sedação e relaxamento muscular adequado, aliado a mínimas alterações sistêmicas. Dentre os tranqüilizantes, as fenotiazinas são possuidoras dessas características, sendo largamente utilizadas em Medicina Veterinária como pré-anestésicos, apresentando ainda efeitos diversos como ação sedativa, adrenolítica e ansiolítica (MASSONE, 1999).

A levomepromazina, fármaco pertencente a esse grupo, com ação predominantemente sedativa, destaca-se pelas características antiarrítmica e antihistamínica, proporcionando sedação em cães por aproximadamente duas a três horas (GROSS, 2003). Produz hipotonia muscular com sonolência, propiciando tranqüilização agradável e segura, além de possuir propriedades úteis como ansiolítica, antiespasmódica e antiemética (ZANINI \& OGA, 1994; MASSONE, 1999). Esta fenotiazina causa depressão no sistema nervoso central (SNC) por ação nos centros nervosos subcorticais, no tálamo, no hipotálamo e pela formação reticular (MASSONE, 1999). A levomepromazina inibe a transmissão sensorial pela diminuição da concentração de dopamina e serotonina na região do gânglio da base (BEDNARSK, 1992), reduzindo o comportamento agressivo e promovendo relaxamento muscular ao deprimirem o sistema reticular ativador no tronco cerebral (AHRENS, 1997).

No sistema cardiovascular, a levomepromazina determina bloqueio $\alpha$-adrenérgico dose dependente, podendo causar hipotensão com taquicardia reflexa pela diminuição da regulação vasomotora (ZANINI \& OGA, 1994). Os antagonistas $\alpha_{1}$-adrenérgicos induzem hipotensão, que é contrabalançada pela atuação reflexa dos barorreceptores, que elevam a freqüência cardíaca (FC), o débito cardíaco (DC) e aumentam a retenção de líquidos (HOFFMAN \& LEFKOWITZ, 1996). PUDDU et al. (1985) concluíram que o emprego da levomepromazina como medicação pré-anestésica diminui a incidência de fibrilação ventricular durante as cirurgias cardíacas em cães. Nesse sentido, ZANINI \& OGA (1994) atribuíram à estabilidade cardíaca promovida pela levomepromazina uma ação análoga à da quinidina. Segundo NUNES et al. (1999), o agente pode ser indicado para controle de complexos ectópicos ventriculares induzidos pelas catecolaminas endógenas e exógenas.
O butorfanol, derivado sintético da morfina, é um opióide sedativo e analgésico, com propriedades agonista e antagonista (HOSGOOD, 1990). A ação antagonista do butorfanol diferencia-o dos opióides tradicionais e da morfina, que apresenta atividade exclusivamente agonista (AHRENS, 1997). Nesse sentido, os opióides sintéticos foram desenvolvidos em função da identificação farmacológica de seus receptores (BEDNARSKI \& MUIR, 1998), que, mediante o mecanismo da interação com um ou mais receptores, produzem sedação com analgesia sem excitação e discreta depressão respiratória (HOSGOOD, 1990; AHRENS, 1997).

No sistema cardiovascular, o butorfanol causa discreta hipotensão por promover relaxamento da musculatura vascular periférica, diminuindo a pressão arterial diastólica e, conseqüentemente, reduzindo a pressão arterial média (PAM) (TRIM, 1983). O fármaco promove aumento transitório da freqüência cardíaca e, ocasionalmente, observa-se arritmia sinusal após a administração intravenosa, seguida de discreta bradicardia, sem contudo alterar o débito cardíaco e a pressão arterial pulmonar (MUIR, 1991). Entretanto, HOUGHTON et al. (1991) não encontraram alterações significativas na PAM e na FC, em cães, após administração IV desse agente na dose de $0,4 \mathrm{mg} \mathrm{kg}^{-1}$. Segundo SHORT et al. (1987), os anticolinérgicos, como o sulfato de atropina, podem ser utilizados para prevenção da bradicardia promovida pelo uso do butorfanol.

Estudo realizado por TRIM (1983) demonstrou que o butorfanol interfere pouco nos parâmetros ventilatórios. O fármaco diminui a freqüência respiratória $(f)$, aumentando discretamente a pressão parcial de $\mathrm{CO}_{2}\left(\mathrm{PaCO}_{2}\right)$ e diminuindo a pressão parcial de $\mathrm{O}_{2}\left(\mathrm{PaO}_{2}\right)$, se administrado IV, em cães, na dose de $0,4 \mathrm{mg} \mathrm{kg}^{-1}$. Entretanto, TRANQUILLI et al. (1988) observaram que a administração da associação de acepromazina e cetamina pela via intramuscular (IM), precedida de butorfanol, em gatos, resultou em depressão respiratória intensa.

O efeito analgésico produzido pelo butorfanol resulta da sua interação com vários sítios de ligação presentes no SNC (ORSINI, 1988). Para estímulos dolorosos superficiais, o efeito analgésico foi evidente em eqüinos 15 minutos após a aplicação de butorfanol IM (ORSINI, 1988). A hiperpolarização neuronal é o provável mecanismo de bloqueio de liberação de neurotransmissores nas vias neuronais excitatórias (REIZINE \& PASTERNAK, 1996). Em relação à analgesia visceral, o butorfanol se mostra mais potente que a morfina, mas, para a analgesia superficial, possui efeito analgésico discreto (MUIR, 
1991). SAWYER et al. (1991) observaram que o tempo máximo de analgesia visceral varia de 23 a 53 minutos, quando o butorfanol é utilizado por via IM, na dose de $0,2 \mathrm{mg} \mathrm{kg}^{-1}$, em cães. Entretanto, quando empregado no período pós-operatório, a analgesia varia entre 55 e 85 minutos. Segundo estudo realizado por HOUGHTON et al. (1991), o butorfanol pode ser utilizado sem estar associado a outros fármacos anestésicos, produzindo analgesia visceral por 30 minutos, na dose de $0,4 \mathrm{mg}$ $\mathrm{kg}^{-1}$, IV, em cães, com período de sedação superior a 75 minutos.

Estudos farmacocinéticos do butorfanol em cães demonstraram que ele é rapidamente absorvido e distribuído no organismo quando injetado IM, na dose de 0,2 a $0,4 \mathrm{mg} \mathrm{kg}^{-1}$, apresentando efeito sedativo 15 minutos após a aplicação, com analgesia clínica de duas horas e efeito antitussígeno acima de quatro horas. Sua ação tem início seis minutos após aplicação IM, com efeito analgésico máximo após 30 minutos. O fármaco tem ação imediata após aplicação IV, sendo grande parte do princípio ativo biotransformado pelo fígado e a maior parte eliminada pelos rins (HOSGOOD, 1990). A dose comumente empregada em cães é de 0,2 a $0,4 \mathrm{mg} \mathrm{kg}^{-1}$ e pode-se administrar o butorfanol por via intravenosa, intramuscular e subcutânea (GWENDOLYN \& CARROLL, 1996). O período de latência foi determinado por KOJIMA et al. (1999) como sendo ao redor de 16 minutos e sua duração de ação em torno de 4 horas, em cães (FANTONI \& MASTROCINQUE, 2002).

Desta forma, com este experimento objetivou-se avaliar os efeitos da administração do butorfanol em cães pré-tratados ou não pela levomepromazina sobre parâmetros circulatórios e respiratórios.

\section{MATERIAL E MÉTODOS}

Foram utilizados 20 cães adultos, machos e fêmeas, sem raça definida, considerados sadios após avaliação clínica e laboratorial, fornecidos pelo canil do Hospital Veterinário “Governador Laudo Natel” da Faculdade de Ciências Agrárias e Veterinárias - FCAV/ UNESP, Câmpus de Jaboticabal, SP. Os animais foram distribuídos aleatoriamente em dois grupos de 10 animais cada, GC (7,5 $\pm 3,0 \mathrm{~kg})$ e GL $(10,3 \pm 4,2 \mathrm{~kg})$.

Aos animais do GC foi administrado IV, $0,2 \mathrm{ml}$ $\mathrm{kg}^{-1}$ de solução salina a 0,9\% (controle) e, após 15 minutos, foi administrado pela mesma via butorfanol ${ }^{\mathrm{a}}$ na dose de $0,3 \mathrm{mg} \mathrm{kg}^{-1}$. Em seguida, os animais foram posicionados em decúbito lateral esquerdo. Nos animais do GL, foi empregada a mesma metodologia, substituindo-se a solução salina a $0,9 \%$ pela levomepromazina ${ }^{\mathrm{b}}$ na dose de $1 \mathrm{mg} \mathrm{kg}^{-1}$, administrada pela mesma via e em volume equivalente ao que seria utilizado de solução salina.

Foram avaliados os valores de freqüência cardíaca (FC), em batimentos por minuto, empregandose eletrocardiógrafo computadorizado ${ }^{c}$ ajustado para leitura na derivação DII; de pressões arteriais sistólica, diastólica e média (PAS, PAD e PAM, respectivamente), em mmHg, por meio de monitor multiparamétrico ${ }^{\mathrm{d}}$ não invasivo, tipo oscilométrico, cujo manguito, de tamanho proporcional ao diâmetro dos membros dos animais, foi adaptado ao membro torácico direito, acima da articulação do cotovelo, sobre a artéria braquial; de freqüência respiratória (f), em movimentos por minuto, e concentração de dióxido de carbono ao final da expiração $\left(\mathrm{ETCO}_{2}\right)$, em mmHg, obtidas por leitura direta em oxicapnógrafo ${ }^{\mathrm{e}}$, adaptando-se o sensor à saída da máscara facial; de saturação de oxihemoglobina $\left(\mathrm{SpO}_{2}\right)$, em porcentagem, obtida por leitura direta em oxicapnógrafo, sendo o emissor/sensor adaptado em região cutânea compatível com a sensibilidade do dispositivo (lábios, orelhas, prepúcio ou mamas); de volume corrente (VC), em mL e volume minuto (VM), em $m L$ por minuto, empregando-se ventilômetro digital ${ }^{\mathrm{f}}$ dotado de sensor, que foi adaptado à saída da máscara facial vedada.

As mensurações foram realizadas imediatamente antes da aplicação dos fármacos (M1). Novas mensurações foram realizadas 15 minutos após a administração da solução salina a $0,9 \%$ ou da levomepromazina (M2) e 10 minutos após a administração do butorfanol (M3). As demais observações foram realizadas a intervalos de 10 minutos, durante 30 minutos (M4, M5 e M6, respectivamente).

A avaliação estatística das variáveis cardiorrespiratórias foi efetuada por meio de Análise de Variância (ANOVA), seguida pelo teste de Tukey $(\mathrm{P}<0,05)$, para as comparações das médias ao longo do tempo em cada grupo e entre os grupos a cada momento.

\section{RESULTADOS}

A freqüência cardíaca não apresentou diferenças significativas entre os grupos, tampouco no grupo que recebeu solução salina a $0,9 \%$. Entretanto, no grupo tratado com levomepromazina, as médias de M1 e M2 foram maiores que as demais. Na avaliação das pressões arteriais, a PAS e a PAM não apresentaram alterações significativas, enquanto que a PAD apresentou média em M5 menor que a de M1 no grupo ao qual foi administrada solução salina a 0,9\% (Tabela 1).

Relativamente à freqüência respiratória, as médias de M5 e M6 foram significativamente menores 
Tabela 1 - Valores médios e desvios padrão $(\mathrm{x} \pm \mathrm{s})$ das variáveis: freqüência cardíaca (FC), pressões arteriais sistólica, diastólica e média (PAS, PAD e PAM), freqüência respiratória (f), concentração de dióxido de carbono ao final da expiração $\left(\mathrm{ETCO}_{2}\right)$, saturação da oxihemoglobina $\left(\mathrm{SpO}_{2}\right)$, volume corrente (VC) e volume minuto (VM) obtidos em cães tratados com solução salina a 0,9\% e butorfanol (GC) ou levomepromazina e butorfanol (GL).

\begin{tabular}{|c|c|c|c|c|c|c|c|}
\hline Variáveis & & M1 & M2 & M3 & M4 & M5 & M6 \\
\hline \multirow{2}{*}{ FC (bat./min.) } & GC & $97 \pm 13$ & $95 \pm 11$ & $89 \pm 9$ & $89 \pm 9$ & $85 \pm 8$ & $87 \pm 13$ \\
\hline & GL & $106 \pm 16^{\mathrm{a}}$ & $110 \pm 20^{\mathrm{a}}$ & $84 \pm 12^{b}$ & $79 \pm 13^{b}$ & $79 \pm 19^{b}$ & $81 \pm 18^{b}$ \\
\hline \multirow{2}{*}{ PAS (mmHg) } & GC & $120 \pm 17$ & $111 \pm 18$ & $110 \pm 18$ & $112 \pm 18$ & $104 \pm 18$ & $120 \pm 29$ \\
\hline & GL & $114 \pm 14$ & $120 \pm 21$ & $110 \pm 20$ & $108 \pm 16$ & $114 \pm 18$ & $115 \pm 15$ \\
\hline \multirow{2}{*}{ PAD (mmHg) } & GC & $81 \pm 14^{\mathrm{a}}$ & $72 \pm 21$ & $65 \pm 15$ & $68 \pm 14$ & $59 \pm 16^{\mathrm{b}}$ & $64 \pm 18$ \\
\hline & GL & $70 \pm 13$ & $65 \pm 14$ & $66 \pm 15$ & $59 \pm 10$ & $60 \pm 6$ & $65 \pm 11$ \\
\hline \multirow{2}{*}{ PAM (mmHg) } & GC & $96 \pm 15$ & $85 \pm 18$ & $80 \pm 14$ & $84 \pm 14$ & $74 \pm 19$ & $84 \pm 22$ \\
\hline & GL & $86 \pm 13$ & $85 \pm 17$ & $82 \pm 17$ & $78 \pm 10$ & $80 \pm 9$ & $84 \pm 11$ \\
\hline \multirow{2}{*}{$f$ (mov./min.) } & GC & $15 \pm 6$ & $15 \pm 6$ & $14 \pm 6$ & $14 \pm 8$ & $15 \pm 7$ & $14 \pm 9$ \\
\hline & GL & $18 \pm 6^{\mathrm{a}}$ & $15 \pm 5$ & $13 \pm 7$ & $11 \pm 5$ & $10 \pm 3^{b}$ & $9 \pm 2^{b}$ \\
\hline \multirow{2}{*}{$\mathrm{ETCO}_{2}(\mathrm{mmHg})$} & GC & $33 \pm 4$ & $33 \pm 4$ & $33 \pm 5$ & $33 \pm 5$ & $32 \pm 4$ & $32 \pm 4$ \\
\hline & GL & $34 \pm 1$ & $32 \pm 2$ & $32 \pm 4$ & $33 \pm 3$ & $34 \pm 3$ & $33 \pm 3$ \\
\hline \multirow{2}{*}{$\mathrm{SpO}_{2}(\%)$} & GC & $97,0 \pm 0,9$ & $95,3 \pm 1,5$ & $94,5 \pm 1,8$ & $95,1 \pm 2,0$ & $94,6 \pm 1,7$ & $95,0 \pm 1,6$ \\
\hline & GL & $97,4 \pm 0,8^{\mathrm{a}}$ & $97,1 \pm 1,2^{\mathrm{a}}$ & $95,8 \pm 2,2$ & $94,5 \pm 2,6^{\mathrm{b}}$ & $95,2 \pm 2,4$ & $95,8 \pm 1,3$ \\
\hline \multirow{2}{*}{$\mathrm{VC}(\mathrm{mL})$} & GC & $295 \pm 105$ & $214 \pm 90$ & $193 \pm 72$ & $184 \pm 49$ & $229 \pm 90$ & $203 \pm 73$ \\
\hline & GL & $261 \pm 82$ & $240 \pm 78$ & $211 \pm 83$ & $228 \pm 88$ & $200 \pm 52$ & $229 \pm 179$ \\
\hline \multirow{2}{*}{ VM (mL/min.) } & GC & $4025 \pm 1264$ & $3133 \pm 1406$ & $2585 \pm 1047$ & $2375 \pm 1010$ & $3303 \pm 1622$ & $2858 \pm 1732$ \\
\hline & GL & $4691 \pm 1671^{\mathrm{a}}$ & $3602 \pm 1634$ & $2465 \pm 979^{b}$ & $2502 \pm 1122^{b}$ & $1877 \pm 561^{\mathrm{b}}$ & $2045 \pm 1617^{b}$ \\
\hline
\end{tabular}

Médias seguidas por letras diferentes nas linhas diferem significativamente entre si pelo teste de Tukey $(\mathrm{P}<0,05)$.

que a média de M1 no grupo tratado pela levomepromazina. Quanto ao $\mathrm{CO}_{2}$ ao final da expiração, não houve variação desse parâmetro entre os grupos, assim como entre os momentos para cada grupo separadamente. Na saturação de oxihemoglobina, a média de M4 foi significativamente menor que as de M1 e M2, no GL. Sobre a ventilometria, o volume corrente (VC) não apresentou diferenças significativas e, no volume minuto (VM), observou-se que a média de M1 foi maior que as médias de M3, M4, M5 e M6, no GL (Tabela 1).

\section{DISCUSSÃO}

A ausência de alteração da FC ao longo dos momentos no GC foi semelhante ao descrito por HOUGHTON et al. (1991) e SAWYER et al. (1991), empregando diversas doses (0,025, 0,05, 0,1, 0,2 e 0,4mg $\mathrm{kg}^{-1}$ ) de butorfanol em cães, e por STICK et al. (1989) e MUIR (1991), em experimentos com eqüinos. Entretanto, o butorfanol poderia produzir discreto efeito parassimpatomimético, segundo os estudos realizados por TRIM (1983), GREENE et al. (1990) e QUANDT et al. (1994), que descreveram a ocorrência de bradicardia em cães que receberam $0,4 \mathrm{mg} \mathrm{kg}^{-1}$ pela via IV.É possível que a ausência de alterações nos batimentos cardíacos esteja relacionada com a dose de butorfanol utilizada, menor que a empregada por TRIM (1983), GREENE et al. (1990) e QUANDT et al. (1994).

No GL, houve diminuição na FC nos momentos M3, M4, M5 e M6, fato esse que corrobora os achados de CORNICK \& HARTSFIELD (1992), que administraram butorfanol associado a um fenotiazínico em cães. A bradicardia encontrada poderia ser atribuída aos efeitos adrenolíticos da levomepromazina, associados ao aumento da atividade vagal (BOVILL et al., 1984) exercida pelo opióide. A excitabilidade elétrica do miocárdio, a freqüência de condução ou a geração de estímulos elétricos no nódulo sinusal poderiam ser inibidos pelos efeitos depressivos da associação dos fármacos.

Relativamente às pressões arteriais, nos estudos realizados por TRIM (1983), HOUGHTON et al. (1991) e SAWYER et al. (1991) em cães, o uso do butorfanol não causou alterações na PAS, na PAD e na PAM, nas doses de 0,2 a $0,4 \mathrm{mg} \mathrm{kg}^{-1}$. A exemplo deles, valores para a PAS, nos dois grupos, não variaram, possivelmente pela ação dos mecanismos compensatórios responsáveis pela manutenção da pressão arterial, como a ativação dos quimio e barorreceptores e do sistema renina-angiotensina e 
aldosterona. Entretanto, MATTHEWS (1987) e QUANDT et al. (1994), empregando o butorfanol em cães na dose de $0,4 \mathrm{mg} \mathrm{kg}^{-1}$, observaram diminuição temporária da PAS. É provável que o volume sangüíneo venoso que retorna aos átrios direito e esquerdo esteja diminuído devido à vasodilatação periférica, reduzindo, portanto, o volume de preenchimento das câmaras cardíacas, causando diminuição da PAD e da PAM. JAFFE \& MARTIN (1985) e STICK et al. (1989) concluíram que os opióides alteram a função do sistema cardiovascular pelo estímulo dos quimioreceptores periféricos e centrais, que podem tanto aumentar como diminuir a PA. MUIR(1991) eAHRENS (1997) atribuíram a diminuição da PAM ao uso do butorfanol, em eqüinos, devido a sua ação agonista sobre os receptores opióides “delta” na região do núcleo solitário no SNC e por promover vasodilatação periférica.

A ausência de alterações significativas no GL nos valores da PAS, da PAD e da PAM diferiram do encontrado por CORNICK \& HARTSFIELD (1992), com a associação entre butorfanol e acepromazina. Segundo os autores, a hipotensão foi devida à somatória dos efeitos vasodilatadores dos fármacos empregados sobre a vasculatura periférica. É possível que o efeito hipotensivo proveniente da vasodilatação periférica tenha sido neutralizado pela ação da cascata reninaangiotensina e aldosterona e pela ativação do sistema nervoso simpático, liberando catecolaminas na circulação em quantidade suficiente para equilibrar as possíveis alterações das pressões arteriais. Por outro lado, considerando-se o fato de que os valores relativos à PAS, à PAD e à PAM foram colhidos 10 minutos após a administração do butorfanol, é possível que tenha ocorrido hipotensão temporária durante esse intervalo, recuperando os valores normais, posteriormente, após ativação dos mecanismos compensatórios, o que corrobora os achados de DYSON (1990) e QUANDT et al. (1994), os quais observaram hipotensão transitória 5 minutos após administração de butorfanol em cães anestesiados com halotano.

Segundo THURMON et al. (1996) e AHRENS (1997), a diminuição da freqüência respiratória é freqüente na associação de fenotiazínicos com opióides na maioria das espécies. Estudos feitos em eqüinos por MUIR (1991) demonstraram que a depressão respiratória seria provocada pela diminuição da atividade do tronco cerebral. É possível que a diminuição da $f$ seja resultado da somatória dos efeitos depressores da levomepromazina e do butorfanol no SNC e pela ação agonista do butorfanol sobre os receptores "delta" localizados no bulbo e nos centros pontinos (MUIR, 1991). Segundo THURMON et al. (1996), sedativos combinados com analgésicos opióides modificam o ritmo respiratório pela alteração no controle neural central da respiração e pela alteração da atividade dos quimiorreceptores. Estudos feitos por REIZINE \& PASTERNAK (1996), demonstraram que a maioria dos opióides tem seus efeitos depressores respiratórios prolongados e exacerbados pelas fenotiazinas, devido à diminuição da atividade dopaminérgica. Dessa forma, é provável que a sensibilização dos subtipos de receptores opióides localizados no SNC exercida pelo butorfanol e o efeito da associação dos fármacos sobre os receptores dopaminérgicos estejam contribuindo para a produção da depressão respiratória.

Relativamente ao $\mathrm{ETCO}_{2}$, não ocorreram alterações significativas em ambos os grupos. Tais resultados foram semelhantes aos encontrados por TRIM (1983), MATTHEWS (1987) e WATERMAN et al. (1991) utilizando o butorfanol. CORNICK \& HARTSFIELD (1992) empregaram o butorfanol em cães pré-tratados com fenotiazínicos e observaram que a combinação deprime os centros respiratórios, porém sem atuar sobre as respostas do organismo ao aumento da concentração de $\mathrm{CO}_{2}$ na circulação.

Com relação à variável $\mathrm{SpO}_{2}$, esta nos permite, de maneira não invasiva e contínua, verificar a qualidade da oxigenação por meio da saturação das hemoglobinas no sangue arterial $\left(\mathrm{SpO}_{2}\right)$. Embora tenham ocorrido alterações significativas ao longo do período experimental no GL, os valores da variável em questão encontram-se dentro dos limites fisiológicos aceitáveis para a espécie. Segundo AHRENS (1997), os opióides combinados com fenotiazínicos para produzir neuroleptoanalgesia estão relacionados com a depressão respiratória devido à somatória dos efeitos depressores ao SNC. Além disso, outro fator importante a ser comentado refere-se ao sensor do oxímetro, o qual foi posicionado em locais variados, nos quais o pulso pôde ser detectado, de tal modo que a pigmentação dos tecidos nesses locais também poderia interferir na qualidade dos sinais obtidos, produzindo assim, de maneira equivocada, diferença estatística significativa em seus valores.

O GC não apresentou alterações significativas na $\mathrm{SpO}_{2}$ ao longo do protocolo experimental, corroborando os achados de WATERMAN et al. (1991), que não observaram alterações nessa variável após administração de $0,2 \mathrm{mg}$ $\mathrm{kg}^{-1}$ de butorfanol em ovinos, e de MATTHEWS (1987), em cães. Por sua vez, TRIM (1983) constatou diminuição nos valores de $\mathrm{SpO}_{2}$ após a administração de $0,4 \mathrm{mg} \mathrm{kg}^{-1}$ de butorfanol por via intravenosa em cães. Provavelmente, os efeitos depressores do agente sobre o SNC tenham ocasionado alterações temporárias na ventilação e no fluxo sangüíneo pulmonar, 
promovendo diminuição da captação adequada de oxigênio para a circulação. Uma possível redução temporária do débito cardíaco pela ação vasomotora do butorfanol ou devido à administração do butorfanol IV realizada de maneira rápida (TRIM, 1983) pode ter ocasionado diminuição da $\mathrm{SpO}_{2}$ nos referidos momentos.

Segundo LUMB \& JONES (1984) e GRANDY \& STEFFEY (1985), a investigação da mecânica respiratória pode ser realizada com ventilômetros adequados para detecção do volume corrente e do volume minuto nos animais. A ventilometria mensura a quantidade de ar que entra e sai dos pulmões; dessa forma, os valores médios de ventilometria obtidos nos momentos estudados para GC e GL apresentaram-se dentro dos limites mínimos aceitáveis para cães, que, segundo SHORT et al. (1987), são de 10 a $20 \mathrm{ml} \mathrm{kg}^{-1}$ para essa espécie.

Para a variável VC, tanto no grupo GC quanto no GL não foram observadas alterações estatísticas significativas ao longo dos momentos. Igual resultado obteve QUANDT et al. (1994) e TRONCY et al. (1996), empregando o butorfanol associado ao halotano em cães. Entretanto, resultados diferentes foram obtidos por MUIR (1991) com a associação de butorfanol e fenotiazínicos, constatando-se diminuição significativa para essa variável em eqüinos. Em ensaios farmacológicos recentes, evidenciou-se a existência de vários subtipos de receptores opióides kappa, como $\mathrm{k}_{1}$ $\mathrm{k}_{2}$ e $\mathrm{k}_{3}$, e subtipos sigma e sigma no SNC, que, após serem estimulados, provavelmente potencializariam os efeitos depressores no centro respiratório. Contudo, o mecanismo de ação dos subtipos de receptores requer ainda melhor compreensão dos efeitos produzidos sobre o SNC (REISINE \& PASTERNAK, 1996). Por outro lado, é possível que mecanismos compensatórios, como aumentos da amplitude ou da complacência da caixa torácica durante os movimentos respiratórios, tenham sido responsáveis pela estabilidade ventilatória.

Quanto ao volume minuto, observou-se diminuição estatística significativa ao longo dos momentos de M3 a M6 no GL, provavelmente causada pela redução da freqüência respiratória ao longo dos respectivos momentos. Segundo MUIR (1991), a associação de butorfanol com tranqüilizantes fenotiazínicos causa depressão no SNC, promovendo diminuição do VM, corroborando os achados obtidos neste experimento. $\mathrm{O}$ conceito de um único centro respiratório é insustentável devido à multiplicidade de fatores e de partes do cérebro que podem influenciar sobre a atividade respiratória. As áreas envolvidas na dinâmica respiratória estão situadas na ponte e no bulbo, porém os centros bulbares possuem conexão com regiões mais altas, como o sistema reticular ativador e o hipotálamo (BURNS \& SALMOIRAGHI, 1960), que possivelmente estariam deprimidos pela ação potencializadora da associação dos fármacos, ocasionando diminuição dos valores médios das variáveis respiratórias.

\section{CONCLUSÃO}

O emprego do butorfanol precedido ou não pela levomepromazina produziu diminuições nas freqüências cardíaca e respiratória, assim como na saturação da oxihemoglobina e no volume minuto. Entretanto, essas alterações foram discretas, não prejudicando os parâmetros circulatórios e respiratórios.

\section{FONTESDEAQUISIÇÃO}

a Torbugesic - Fort Dodge Saúde Animal Ltda. - Campinas, SP, Brasil.

b Neozine - Rhodia S.A. Divisão Farmacêutica - São Paulo, SP, Brasil.

c TEB - mod. ECGPC software versão 1.10 - São Paulo, SP, Brasil.

d Digimax 5000 - mod. ESFMN 2T - São Paulo, SP, Brasil.

e Dixtal - mod. DX7100 - Manaus, AM, Brasil.

${ }^{f}$ VENTCARE. Takaoka Ind. e com LTDA. São Paulo, SP. Brasil.

\section{REFERÊNCIAS}

AHRENS, F.A. Anestésicos. In:_____- Farmacologia veterinária. São Paulo: Artes Médicas, 1997. p.94-98.

BEDNARSK, R.M. Chemical restraint of the standing horse. In: ROBSON, N.E. Current therapy in equine medicine. 3.ed. Philadelphia: Saunders, 1992. p.22-23.

BEDNARSKI, R.M.; MUIR III, W.W. Considerações anestésicas na cirurgia. In: SLATER, D. Manual de cirurgia de pequenos animais. 2.ed. São Paulo: Manole, 1998. p.2645-2652.

BOVILL, J.G. et al. Opioid analgesics in anesthesia: with special reference to their use in cardiovascular anesthesia. Anesthesiology, v.61, p.731-755, 1984.

BURNS, B.D.; SALMOIRAHI, G.C. Repetitive firing of respiratory neurones during their burst activity. J Neurophy, v.23, n.27, p.22, 1960.

CORNICK, J.L.; HARTSFIELD, S.M. Cardiopulmonary and behavioral effects of combinations of acepromazine/butorphanol and acepromazine/oxymorphone in dogs. J Am Vet Med Assoc, v.200, n.12, p.1952-1956, 1992.

DYSON, D.H. Update on butorphanol tartrate: use in small animals. Can Vet J, v.13, n.2, p.120-121, 1990.

FANTONI, D.T.; MASTROCINQUE, S. Fisiopatologia e controle da dor. In: FANTONI, D.T.; CORTOPASSI, S.R. 
Anestesia em cães e gatos. São Paulo: Roca, 2002. p.323336.

GRANDY, J.L.; STEFFEY, E.P. Anesthesia and the respiratory system. In: SLATTER. Textbook of small animal surgery. Philadelphia: Saunders, 1985. Cap.189, p.2621-2633.

GREENE, S.A. et al. Cardiovascular effects of butorphanol in halothane anesthetized dogs. Am J Vet Res, v.51, n.8, p.12761279,1990

GROSS, M.E. Tranqüilizantes, agonistas $\alpha_{2}$ - adrenérgicos e agentes relacionados. In: ADAMS, H.R. Farmacologia e terapêutica em veterinária. 8.ed. Rio de Janeiro: Guanabara Koogan, 2003. Cap.14, p.257.

GWENDOLYN, L.; CARROLL, M.S. How to manage perioperative pain. Vet Med, n.4, p.353-357, 1996.

HOFFMAN, B.B.; LEFKOWITZ, R.J. Catecolaminas, drogas simpaticomiméticas e antagonistas dos receptores adrenérgicos In: GOODMAN, L.S.; GILMAN, A.G. As bases farmacológicas da terapêutica. 8.ed. Rio de Janeiro: Guanabara Koogan, 1996. p.146-178.

HOSGOOD, G. Farmacologia fatures of butorphanol in dogs and cats: topics in drug therapy. J Am Vet Med Assoc, v.196, n.1, p.135-136, 1990.

HOUGHTON, K.J. et al. Dose-response of intravenous butorphanol to increase viceral noceptive threshold in dogs. Soc Exp Biol Med, v.197, p.290-296, 1991.

JAFFE, J.H.; MARTIN, W.R. Opioids analgesics and antagonists. In: GOODMAN, L.S.; GILMAN, A.G. The pharmacological basis of therapeutics. 7.ed. New York: MacMillan, 1985. p.491-531.

KOJIMA, K. et al. Comparison of sedative effects of medetomidine-midazolam, acepromazine-butorphanol and midazolam-butorphanol in dogs. Zentralbl Veterinarmed A, v.46, n.3, p.141-148, 1999.

LUMB, W.V.; JONES, E.W. Veterinary anesthesia. 2.ed. Philadelphia: Lea \& Febiger, 1984. p.663.

MASSONE, F. Medicação pré-anestésica. Anestesiologia veterinária. 3.ed. Rio de Janeiro: Guanabara Koogan, 1999. p. 20-21

MATTHEWS, N.S. Celiling effect for respiratory depression by butorphanol with halothane anesthesia. Vet Surg, v.16, n.4, p.323, 1987.

MUIR, W.W. Standing chemical restraint in horses: tranquilizers, sedatives, and analgesics. In: MUIR, W.W.; HUBBELL, J.A.E
Equine anesthesia: monitoring and emergency therapy. St. Louis: Mosby, 1991. Chap.11, p.247-276.

NUNES, N. et al. Atividade antiarritmogênica da levomepromazina em cães submetidos à anestesia pela quetamina. Ciênc Rural, v.29, n.2, p.291-295, 1999.

ORSINI, J.A. Butorphanol tartrate: pharmacology and clinical indications. Comp Equine, v.10, n.7, p.849-854, 1988.

PUDDU, P.E. et al. Une tecnique d'anesthèsie du chien pour l'étude des effets arythmogène de l'occlusuon coronaire aigue. J Physiol, v.80, n.2, p.27-31, 1985

QUANDT, J.E. et al. Butorphanol does not reduce the minimum alveolar concentration of halotano in dogs. Vet Anesth, v.23, n.2, p.156-159, 1994

REIZINE, T.; PASTERNAK, G. Analgésicos e antagonistas opióides. In: GOODMAN, L. S.; GILMAN, A.G. As bases farmacológicas da terapêutica. 8.ed. Rio de Janeiro: Guanabara Koogan, 1996. p.380-404.

SAWYER, D.C. et al. Dose response to butorphanol administered subcutaneously to increase visceral nociceptive threshold in dogs. Am J Vet Res, v.52, n.11, p.1826-1830, 1991

SHORT, C.E. et al. Preanesthetic and postoperative pain relief in dogs: comparing analgesics. Vet Med, v.6, n.7, p.744-751, 1987

STICK, J.A. et al. Effects of butorphanol tartrate on arterial pressure, jejunal blood flow, vascular resistance, $\mathrm{O}_{2}$ extraction, and $\mathrm{O}_{2}$ uptake in halothane-anesthetized ponies. Am J Vet Res, v.50, n.8, p.1202-1206, 1989.

THURMON, J.C. et al. Preanesthetics and anesthesic adjuncts. In: Lumb \& Jones' veterinary anesthesia. 3.ed Philadelphia: Lea \& Feabiger, 1996. p.186.

TRANQUILLI, W.J. et al. Butorphanol as a preanesthetic in cat: its effects on two common intramuscular regimens. Vet Med, n.8, p.848-853, 1988.

TRIM, C.M. Cardiopulmonary effects of butorphanol tartrate in dogs. Am J Vet Res, v.44, n.2, p.329-331, 1983

TRONCY, E. et al. Evaluation of analgesia and cardiorespiratory effects of epidurally administered butorphanol in isofluraneanesthetized dogs. Am J Vet Res, v.57, n.10, p.1479-1482, 1996.

WATERMAN, A. et al. Analgesic activity and respiratory effects of butorphanol in sheep. Vet Sci, v.51, p.19-23, 1991.

ZANINI, A.C.; OGA, S. Farmacologia aplicada. 5.ed. São Paulo: Atheneu, 1994. p.161-162. 